BMJ Open Diabetes Research \& Care

\title{
Ocular screening adherence across Hispanic/Latino heritage groups with diabetes: results from the Ocular SOL ancillary to the Miami site of the Hispanic Community Health Study/ Study of Latinos (HCHS/SOL)
}

\author{
Stacey L Tannenbaum, ${ }^{1}$ Laura A McClure, ${ }^{1}$ D Diane Zheng, ${ }^{2}$ Byron L Lam, ${ }^{3}$ \\ Kristopher L Arheart, ${ }^{2}$ Charlotte E Joslin, ${ }^{4}$ Gregory A Talavera, ${ }^{5}$ David J Lee ${ }^{1,2,3}$
}

To cite: Tannenbaum SL, McClure LA, Zheng DD, et al. Ocular screening adherence across Hispanic/Latino heritage groups with diabetes: results from the Ocular SOL ancillary to the Miami site of the Hispanic Community Health Study/ Study of Latinos (HCHS/ SOL). BMJ Open Diabetes Research and Care 2016;4: e000236. doi:10.1136/ bmjdrc-2016-000236

- Additional material is available. To view please visit the journal (http://dx.doi.org/ 10.1136/bmjdrc-2016000236).

Received 1 April 2016 Revised 9 June 2016 Accepted 14 July 2016

\section{CrossMark}

For numbered affiliations see end of article.

Correspondence to Dr Laura A McClure; LMcClure@miami.edu

\section{ABSTRACT}

Objective: The objective of this study was to examine the prevalence and correlates of ocular screening adherence among select Hispanics/Latinos living with diabetes.

Research design and methods: Data were obtained through an ancillary study of the Hispanic Community Health Study/Study of Latinos (Miami site).

Participants included Hispanics/Latinos aged 40+ years who underwent a baseline examination/risk factor assessment (2008-2011) and then completed a survey on vision health/knowledge (conducted October 2011September 2013; sample $n=1235$; diabetic subsample=264). The dependent variable was having a dilated eye examination within the past 12 months. Covariate candidate selection for entry into sequential multivariable logistic regression models was guided by Anderson's Behavioral Model of Health Services Use and the Behavioral Model for Vulnerable Populations.

Results: Participants aged 65+ were more likely to have dilated eye examinations (OR 2.62, 95\% $\mathrm{Cl} 1.22$ to 5.60) compared with those aged 40-54 years. Participants less likely to have dilated examinations had a high school degree or general educational development (GED) (OR $0.30,95 \% \mathrm{Cl} 0.10$ to 0.96 , compared to no degree) and those currently uninsured or never insured ((OR $0.34,95 \% \mathrm{Cl} 0.14$ to 0.83$)$ and (OR $0.19,95 \% \mathrm{Cl} 0.07$ to 0.51$)$ ) compared to those currently insured. Participants who heard or saw something about eye health from two or more sources (eg, media outlets, doctor's office, relatives/friends) compared to those who reported no sources in the past 12 months were more likely to have a dilated eye examination (OR 2.82, 95\% Cl 1.26 to 6.28).

Conclusions: Lack of health insurance is strongly associated with low screening uptake in Hispanics/ Latinos living with diabetes. Health promotion strategies stressing the importance of annual dilated eye examinations and increasing sources of information on eye health are other potential strategies to increase screening uptake in Hispanics/ Latinos.

\section{Key messages}

- Our results show poor adherence to ocular screening among Hispanics/Latinos with diabetes as well as poor awareness of the disease diabetic retinopathy.

- Lack of health insurance and underinsurance is strongly associated with low screening uptake in Hispanics/Latinos with diabetes.

- Greater exposure to sources of eye health information may be a predictor of having had a dilated eye examination in the past year among Hispanics/Latinos with diabetes.

\section{INTRODUCTION}

The prevalence of diabetes mellitus has increased by roughly $75 \%$ in the past 20 years. ${ }^{1}$ As more American adults live with diabetes, the incidence of complications from this condition is also increasing; these complications include diabetic retinopathy, the risk of which increases with the number of years living with this illness. ${ }^{2}$ Diabetic retinopathy is a leading cause of blindness in the USA and can be particularly devastating if left undiagnosed and untreated. Vision-threatening diabetic retinopathy is preventable through strict blood sugar control; in addition, regular dilated eye examinations are essential to blindness prevention as they identify early disease that is more easily treated and results in better outcomes. ${ }^{2}$ Management through injections, as well as laser and traditional surgery, is also critical to preventing progressive vision loss when diabetic retinopathy is present and may potentially restore vision in those who have sustained related complications. The American Academy of Ophthalmology and 
the American Diabetes Association recommend annual dilated eye examinations for patients with type 1 and type 2 diabetes, while allowing less frequent examinations after several normal readings. ${ }^{3}{ }^{4}$ However, poor awareness of screening guidelines has been demonstrated among individuals living with diabetes with or without a diabetic retinopathy diagnosis. ${ }^{5}$

Hispanics/Latinos overall in the USA have a higher prevalence of diabetes $(16.9 \%)^{6}$ than non-Hispanic whites $(10.2 \%)^{7}$ and a slightly higher prevalence of prediabetes ( $38 \%$ and $35 \%$, respectively), ${ }^{8}$ although there is variability by Hispanic/Latino ethnicity. ${ }^{6}$ Hispanics/ Latinos with diabetes also have a higher prevalence of diabetic retinopathy than African-Americans and non-Hispanic whites. ${ }^{9}{ }^{10}$ Moreover, there is vast cultural heterogeneity among Hispanics/Latinos residing in the USA $^{11}$ including genetic diversity in diabetic retinopathy, ${ }^{10}$ heritage as well as differences in how varying Hispanic/Latino populations process health-related communications given by healthcare providers. ${ }^{12}$

Ocular health, risk factors, and healthcare screening have been shown to impact overall health, functioning, and mortality among Hispanics/Latinos with diabetes. ${ }^{13-19}$ The National Eye Institute has funded several large epidemiologic studies of ocular health in those of Mexican heritage ${ }^{20-23}$ but to date there has not been a comprehensive evaluation of ocular health issues, including screening adherence, to improve the ocular health across all Hispanic/Latino populations with diabetes, and in particular seldom studied heritage groups, for example, Cubans and Central and South American Hispanics/Latinos.

The main objectives of this study were, first, to document the distribution of dilated ocular screening prevalence in less studied Hispanic/Latino heritage groups with and without diabetes; and second, to identify predisposing, enabling, need, and knowledge determinants associated with screening guideline adherence among those with diabetes. ${ }^{34}$

\section{RESEARCH DESIGN AND METHODS Sample}

Data for our study were obtained through an ancillary study of the Hispanic Community Health Study/Study of Latinos (HCHS/SOL). The HCHS/SOL is an ongoing multisite epidemiologic study of prevalence of and risk factors for disease among Hispanics/Latinos (http:// www.cscc.unc.edu/hchs). A detailed depiction of HCHS/ SOL has been previously described. ${ }^{24}$ There are a total of 4087 participants at the HCHS/SOL Miami site, and the ethnic background of the Miami site sample is $57 \%$ Cuban heritage, 26\% Central American heritage, 12\% South American heritage, 2\% Puerto Rican heritage, 2\% Dominican heritage, and $1 \%$ Mexican heritage. Participants in our ancillary study at the HCHS/SOL Miami site included Hispanics/Latinos aged 40 years and older who underwent a baseline clinical examination and risk factors assessment in 2008-2011 and then completed our survey on vision health and knowledge of ocular disease $(n=1235)$. Of eligible Miami sites participants, $43.4 \%$ completed our ocular health survey. This ancillary study, hereafter referred to as the Ocular Study of Latinos (Ocular SOL), was conducted between October 2011 and September 2013 and was comprised of a convenience sample of HCHS/SOL participants not previously enrolled in two other ancillary studies to the HCHS/ SOL. After data collection, Ocular SOL data were merged with HCHS/SOL parent study baseline data to obtain additional demographic, clinical, and health-related variables. This study was approved by the HCHS/SOL publications committee and by the Institutional Review Board of the University of Miami.

\section{Variables}

Unless otherwise noted, we employed variable definitions utilized in previous HCHS/SOL and Ocular SOL publications. ${ }^{625}$ The outcome variable of interest was, 'When was your last complete eye examination, one that included dilating your pupils, where the doctor used bright lights to look into the back of your eyes? This would have made you temporarily sensitive to bright light'. This variable was categorized as having had a dilated eye examination within the past 12 months (yes, no). Participants who were clinically diagnosed with diabetes and reported having had a dilated eye examination in the previous 12 months were considered adherent. ${ }^{3}{ }^{4}$ Our sample was stratified by diabetes status. Diabetes status was clinically determined at the baseline study, and based on the American Diabetes Association definition using serum glucose (controlling for length of fasting), post-oral glucose tolerance test glucose levels (if available), glycosylated hemoglobin percentages, and oral diabetic medication usage, diabetes status was a dichotomous variable (yes, no) ${ }^{26}$

For our models, we used Andersen's Behavioral Model of Health Services Use ${ }^{27}$ and the Behavioral Model for Vulnerable Populations, ${ }^{28}$ both previously and similarly categorized in other HCHS/SOL analyses, ${ }^{25}$ and knowledge of eye care, to determine the selection and retention of relevant covariates in the following categories: (1) predisposing factors (traditional), (2) predisposing factors (vulnerable), (3) enabling factors, (4) need factors, and (5) knowledge factors. Predisposing factors were considered non-modifiable, enabling factors characterized resources of participants, and need factors prodded participants toward healthcare use and behavioral change. Knowledge factors included knowing about eye care, sources of eye health information, diabetes status, and diabetic retinopathy. All five categories of independent variables were evaluated for their influence on adherence to dilated eye examination within the past 12 months.

\section{Traditional predisposing factors}

Independent variables in the category included age (4054, 55-64, 65+ years), gender (male and female), 
Hispanic/Latino heritage (Cuban, Central/South American, Other), marital status (single, married or living with partner, and separated, divorced, or widowed), education ( $<$ high school (HS) degree or general educational development (GED), HS degree or GED, greater than HS degree), and employment (employed, retired and not currently employed, unemployed).

\section{Vulnerable predisposing factors}

These independent variables included place of birth (USA born vs foreign born), years lived in USA $(\leq 5,6-$ 14, 15+ years), and two subscales from a modified version of Short Acculturation Score for Hispanics/ Latinos (SASH): ${ }^{29}$ the SASH Language Use subscale, which assesses respondents' preferred language in various settings, and the SASH Ethnic Social Relations subscale, which assesses the ethnicity of people in respondents' social circles/interactions. The SASH subscales range from 1 (least acculturated) to 4 (most acculturated).

\section{Enabling factors}

These independent variables included health insurance (currently insured, currently uninsured, never insured), vision insurance separate from health insurance (yes, no), income ( $<20000, \$ 20000-\$ 40000,>\$ 40000)$, difficulty communicating with healthcare provider in the past 12 months (yes, no, did not see provider in past 12 months), and inability to get needed healthcare in past 12 months due to cost (yes, no).

\section{Need factors}

These independent variables included self-reported eyesight (excellent/good, fair, poor/very poor), National Eye Institute-Visual Functioning Questionnaire (NEI-VFQ) score $^{30}$ mean SF-12v2 physical health score, ${ }^{31}$ mean SF-12v2 mental health score, ${ }^{31}$ mean of 6 -item neurocognitive score, ${ }^{32}$ mean word fluency score, ${ }^{32}$ mean digital symbol score, ${ }^{32}$ self-reported chronic diseases (none, one, two or more), smoking status (never, former, current), National Institute on Alcohol Abuse and Alcoholism (NIAAA) defined alcohol use (non-drinker, low-risk drinker, at-risk drinker), self-reported compliance with 2008 physical activity guidelines (yes, no), and mean glycosylated hemoglobin. The NEI-VFQ is a composite score comprised of 25 items that assess the impact of self-reported visual health on emotional well-being and social functioning. ${ }^{30}$ The score is an average of the subscales within the 25 items and has a range of 0 (worst eye health) to 100 (best eye health). Self-rated general health from the HCHS/SOL baseline examination was used to calculate the general health subscale of the NEI-VFQ. One item of the vision-related mental health subscale of NEI-VFQ was not included in the calculation ('How much of the time do you worry about your eyesight') as it was omitted from the survey; the mental health subscale was, therefore, averaged over the three existing mental health subscale items. This modification did not affect the total scoring of NEI-VFQ and our NEI-VFQ scores are comparable with that of other studies. ${ }^{23}{ }^{33}$ The SF-12v2 physical and mental health scores are calculated from the 12-item surveys that measure self-reported functional health and well-being. ${ }^{31}$ Scores are norm-based standardizations to a mean of 50 and an SD of 10. They range from 0 (poorest health) to 100 (best health).

\section{Knowledge factors}

There were several independent variables included in the knowledge factors: participant knowledge of having a diabetes diagnosis (yes, if participant answered yes to 'Has a doctor ever said that you have diabetes (high sugar in blood or urine)?' AND if women answered no or there was missing information when asked the follow-up question, 'Was this during pregnancy only?', otherwise, no to knowledge of diabetes diagnosis). Other independent variables were the total number of all sources where individuals could have seen or heard about eye health or disease (specific sources asked to participants are listed in online supplementary table S1), with possible responses categorized as, have not heard or seen anything about eye health or disease, one source, or two or more sources; awareness of diabetic retinopathy (('Have you ever heard of an eye disease called diabetic retinopathy?' (yes, no/not sure)), and mean General Eye Knowledge Summary scale. The General Eye Knowledge Summary scale was created from participants in Ocular SOL who were administered a series of 14 questions about factors that may help prevent eye disease or preserve vision as identified in the research literature. ${ }^{34} 35$ A principal components factor analysis with a varimax (orthogonal) rotation found eight items that loaded onto a single factor (Cronbach's $\alpha$ internal consistency reliability $=0.78$ ). These items were based on the response to the following question: "Which of the following help prevent eye disease and help preserve your vision?'. Responses were 'Seeing an eye doctor regularly', 'Not smoking', 'Controlling blood pressure', 'Controlling cholesterol', 'Wearing sunglasses', 'Eating dark green leafy and orange vegetables', 'Controlling blood sugar', and 'Avoiding becoming diabetic'. All questions had yes, no, or not sure as response options. The 'no' and 'not sure' items were combined. Items were summed to create the scale with scores ranging from 0 to 8 with 8 indicating greater knowledge.

\section{Statistical analysis}

We used SAS V.9.3 (SAS Institute, Cary, North Carolina, USA) for all analyses and adjusted for the survey design. Descriptive analyses of participant data stratified by diabetes status were conducted to produce percent and SE for categorical variables and mean and SE for continuous variables. Multivariable logistic regression analyses with our binary outcome of interest were conducted to produce ORs and $95 \%$ CIs by adding the five categories 
of variables in this block sequential order: (1) predisposing traditional factors, (2) predisposing vulnerable factors, (3) enabling factors, (4) need factors, and (5) ocular knowledge factors. Only variables significant at $\mathrm{p}<0.2$ from each sequential model were retained in the subsequent block such that at model 5 only relevant predictors that were sequentially retained for analysis were presented for the final model; in the final model, only predictors significant at the $\mathrm{p}<0.05$ level were retained. Owing to its clinical importance, we retained age throughout models 1-4 regardless of it breaching the 0.2 cut-off. In addition to being conceptually grounded, this sequential block approach is helpful with small samples as it allows us to evaluate these factors without examining complex models that produce small analytic cell sizes.

\section{RESULTS}

Of the 1235 participants in the Ocular SOL, 264 $(21.4 \%)$ had clinically confirmed diabetes and 971 (78.6\%) did not have diabetes at baseline. Descriptive information of the predisposing (traditional and vulnerable), enabling, need, and knowledge factors of the study sample, stratified by diabetes status, is displayed in table 1 . The majority of participants with diabetes compared to those without diabetes self-reported having a dilated eye examination within the last 12 months $(61.8 \%$ vs $36.5 \%)$. Participants with a diabetes diagnosis were more likely to be aged 65 and older $(41.3 \%$ vs $12.9 \%$ no diabetes), employed ( $26.1 \%$ vs $10.2 \%$ no diabetes), and were more likely to have health insurance $(67.9 \%$ vs $40.1 \%$ no diabetes); few in either group reported having separate vision insurance $(11.5 \%$ vs $6.5 \%$ no diabetes). The majority of participants living with diabetes self-reported two or more chronic diseases (61.1\% vs $13.0 \%$ no diabetes), were not alcohol drinkers (57.4\% vs $39.7 \%$ no diabetes), did not meet 2008 physical activity guidelines ( $56.6 \%$ vs $43.4 \%$ no diabetes), and had not heard of diabetic retinopathy $(53.3 \%$ vs $65.1 \%$ no diabetes). Only $58.7 \%$ of those with a clinically determined diagnosis of diabetes at baseline also selfreported diabetes status at the baseline examination.

Results of all multivariable logistic regression models predicting dilated eye examination in persons with diabetes within the past 12 months are depicted in table 2 . Regression coefficient estimates are presented only for independent variables significant at $\mathrm{p}<0.2$ for the first five models and at the $\mathrm{p}<0.05$ for the final model. In this final model, participants aged 65 years and older were more than 2.5 times as likely to have a dilated eye examination (OR 2.62, 95\% CI 1.22 to 5.60) compared with those aged 40-54 years. Participants less likely to have a dilated eye examination included those with a high school degree or GED (OR $0.3095 \%$ CI 0.10 to 0.96 ) compared to no degree and those currently uninsured or never insured ( (OR, $0.34 ; 95 \%$ CI 0.14 to 0.83 ) and (OR, $0.19 ; 95 \%$ CI 0.07 to 0.51$)$, respectively) compared to those currently insured. Participants who heard or saw something about eye health from two or more sources in the past 12 months were more likely to have a dilated eye examination than those who did not hear or see any information (OR 2.82, 95\% CI 1.26 to $6.28)$.

\section{CONCLUSIONS}

This study builds on our previous broader investigation of healthcare utilization in the same population of Hispanic/Latino heritage groups for the following outcomes: (1) ever had an eye examination, (2) eye examination within the past 2 years, and (3) difficulty getting needed eye care in the past year. ${ }^{25}$ The present multivariable analysis was limited to those living with diabetes. We demonstrated poor adherence with recommended ocular screening among Hispanics/Latinos with diabetes, one of the most important preventive measures against vision loss in diabetic retinopathy. Comparing Hispanics/Latinos with diabetes to those without diabetes, we found that the former group was much more likely to report having a dilated eye examination within the previous 12 months $(61.8 \%$ vs $36.5 \%)$. While the finding that persons with diabetes had higher screening rates is encouraging, the fact that nearly $40 \%$ of those living with diabetes had not met the current diabetic screening guidelines is troubling because early diagnosis of retinal disease is more easily managed and treated than disease found in the later stages.

Furthermore, it should be noted that, with a signed release, selected results of participant baseline examinations were sent to the participants and their designated healthcare provider, which included notification of results suggestive of a diabetes diagnosis. Therefore, the overall post-baseline level of diabetes awareness in our sample theoretically should have increased as an unknown proportion of these participants would have sought follow-up care for their newly discovered diabetes. Moreover, since the follow-up ocular survey took place 1-2 years following the baseline examination, all of these newly diagnosed cases had adequate time to obtain a dilated eye examination. In addition, since $41.3 \%$ of persons who were clinically diagnosed with diabetes were apparently unaware of their disease at baseline, this speaks to a larger issue of general healthcare access and screening.

Post hoc analysis of adherence with dilated eye examination recommendations, stratified by awareness of diabetes diagnosis at baseline, demonstrated that despite being notified of their diabetes status after their baseline examination, only $26.4 \%$ reported a subsequent dilated eye examination by the time of Ocular SOL. This screening rate is far lower than for Ocular SOL participants who were aware of their diabetes status at baseline $(68.7 \%)$. The need for dilated eye examinations in those newly diagnosed is paramount and should be immediately 
Table 1 Prevalence of dilated eye examination in the past 12 months by eye examination history and predisposing, enabling, need, and ocular knowledge factors of Ocular SOL participants by diabetes status

\begin{tabular}{|c|c|c|c|c|c|c|}
\hline & \multicolumn{3}{|c|}{ No diabetes $(\mathrm{N}=971)$} & \multicolumn{3}{|c|}{ Diabetes $(\mathrm{N}=264)$} \\
\hline & $\mathbf{n}$ & $\% /$ Mean & $\mathrm{SE}^{\star}$ & $\mathbf{n}$ & $\% /$ Mean & $\mathbf{S E}^{\star}$ \\
\hline \multicolumn{7}{|l|}{ Last complete dilated eye examination } \\
\hline Within last 12 months & 296 & 36.52 & 2.13 & 135 & 61.80 & 3.54 \\
\hline $1-3$ years ago & 274 & 29.65 & 2.00 & 64 & 22.45 & 3.10 \\
\hline $3-5$ years ago & 103 & 11.31 & 1.22 & 16 & 6.37 & 1.98 \\
\hline$>5$ years ago & 151 & 17.77 & 1.60 & 21 & 6.49 & 1.33 \\
\hline Never & 47 & 4.75 & 0.76 & 7 & 2.89 & 1.12 \\
\hline \multicolumn{7}{|l|}{ Predisposing factors (traditional) } \\
\hline \multicolumn{7}{|l|}{ Age group (years) } \\
\hline $40-54$ & 602 & 61.37 & 2.33 & 89 & 26.81 & 3.35 \\
\hline $55-64$ & 306 & 25.71 & 1.95 & 119 & 31.86 & 3.05 \\
\hline $65+$ & 63 & 12.92 & 1.62 & 56 & 41.33 & 4.38 \\
\hline \multicolumn{7}{|l|}{ Gender } \\
\hline Women & 606 & 54.44 & 1.77 & 151 & 49.48 & 3.80 \\
\hline Men & 365 & 45.56 & 1.77 & 113 & 50.52 & 3.80 \\
\hline \multicolumn{7}{|l|}{ Hispanic Latino background } \\
\hline Cuban & 580 & 72.52 & 2.20 & 168 & 76.27 & 3.32 \\
\hline Central/South American & 329 & 20.35 & 1.75 & 78 & 14.91 & 2.46 \\
\hline Other & 62 & 7.13 & 1.21 & 18 & 8.82 & 2.44 \\
\hline \multicolumn{7}{|l|}{ Marital status } \\
\hline Single & 192 & 18.47 & 1.67 & 44 & 15.69 & 3.32 \\
\hline Married or living with partner & 522 & 56.72 & 2.59 & 150 & 54.55 & 3.99 \\
\hline Separated, divorced, or widowed & 256 & 24.81 & 1.77 & 67 & 29.75 & 3.50 \\
\hline \multicolumn{7}{|l|}{ Educational attainment } \\
\hline No HS degree/GED & 238 & 22.57 & 2.00 & 90 & 34.11 & 3.06 \\
\hline HS degree/GED & 256 & 25.68 & 2.06 & 59 & 21.53 & 2.80 \\
\hline$>\mathrm{HS}$ degree/GED & 477 & 51.75 & 2.24 & 113 & 44.36 & 3.30 \\
\hline \multicolumn{7}{|l|}{ Employment status } \\
\hline Employed & 63 & 10.20 & 1.46 & 45 & 26.08 & 4.31 \\
\hline Retired and not currently employed & 418 & 41.08 & 2.00 & 139 & 46.86 & 3.90 \\
\hline Unemployed & 489 & 48.72 & 1.85 & 79 & 27.06 & 3.58 \\
\hline \multicolumn{7}{|l|}{ Predisposing factors (vulnerable) } \\
\hline \multicolumn{7}{|l|}{ Place of birth } \\
\hline Foreign born & 935 & 95.08 & 1.25 & 256 & 97.83 & 0.94 \\
\hline USA born (including territories) & 36 & 4.92 & 1.25 & 6 & 2.17 & 0.94 \\
\hline \multicolumn{7}{|l|}{ Years lived in USA (among foreign born) } \\
\hline$\geq 5$ & 263 & 30.26 & 2.20 & 52 & 19.72 & 3.12 \\
\hline $6-15$ & 356 & 34.85 & 1.96 & 90 & 32.91 & 4.36 \\
\hline$>15$ & 352 & 34.89 & 2.69 & 122 & 47.37 & 5.07 \\
\hline SASH Language Preference subscale & 970 & 1.40 & 0.026 & 262 & 1.33 & 0.05 \\
\hline SASH Ethnic Social Relations subscale & 889 & 1.97 & 0.023 & 239 & 1.96 & 0.04 \\
\hline \multicolumn{7}{|l|}{ Enabling factors } \\
\hline \multicolumn{7}{|l|}{ Health insurance } \\
\hline Currently insured & 338 & 40.11 & 2.91 & 143 & 67.87 & 3.81 \\
\hline Currently uninsured & 416 & 40.44 & 2.47 & 66 & 18.70 & 2.37 \\
\hline Never been insured & 211 & 19.45 & 1.78 & 50 & 13.43 & 2.88 \\
\hline \multicolumn{7}{|l|}{ Type of health insurance } \\
\hline Medicaid & 27 & 3.91 & 1.11 & 15 & 5.09 & 1.73 \\
\hline Medicare & 166 & 19.68 & 1.93 & 83 & 41.94 & 3.84 \\
\hline Private & 141 & 15.62 & 2.20 & 42 & 19.04 & 3.79 \\
\hline Other insurance & 4 & 0.52 & 0.33 & 3 & 0.98 & 0.58 \\
\hline None & 633 & 60.27 & 2.90 & 121 & 32.95 & 3.90 \\
\hline \multicolumn{7}{|l|}{ Vision insurance (separate from health) } \\
\hline Yes & 52 & 6.52 & 1.32 & 22 & 11.54 & 3.08 \\
\hline No & 913 & 93.48 & 1.32 & 242 & 88.46 & 3.08 \\
\hline
\end{tabular}


Table 1 Continued

\begin{tabular}{|c|c|c|c|c|c|c|}
\hline & \multicolumn{3}{|c|}{ No diabetes $(\mathrm{N}=971)$} & \multicolumn{3}{|c|}{ Diabetes $(\mathrm{N}=264)$} \\
\hline & $\mathbf{n}$ & $\% /$ Mean & SE $^{\star}$ & $\mathbf{n}$ & $\% /$ Mean & $\mathrm{SE}^{\star}$ \\
\hline \multicolumn{7}{|l|}{ Household income } \\
\hline$<\$ 20000$ & 517 & 56.20 & 2.54 & 153 & 64.20 & 4.06 \\
\hline$\$ 20001-\$ 40000$ & 262 & 30.31 & 2.11 & 66 & 29.22 & 3.79 \\
\hline$>\$ 40000$ & 84 & 13.50 & 1.91 & 14 & 6.58 & 2.50 \\
\hline \multicolumn{7}{|c|}{ Difficulty communicating with a healthcare provider in the past 12 months } \\
\hline No & 350 & 37.96 & 2.65 & 145 & 59.34 & 4.33 \\
\hline Yes & 38 & 3.42 & 0.75 & 16 & 5.97 & 1.95 \\
\hline Did not see provider past 12 months & 583 & 58.62 & 2.84 & 103 & 34.69 & 4.54 \\
\hline \multicolumn{7}{|c|}{ Could not get needed healthcare in the previous 12 months because of cost } \\
\hline No & 813 & 83.99 & 1.86 & 228 & 90.76 & 2.10 \\
\hline Yes & 158 & 16.01 & 1.86 & 36 & 9.24 & 2.10 \\
\hline \multicolumn{7}{|l|}{ Need factors } \\
\hline Mean SF-12 Physical Health Score & 953 & 49.88 & 0.44 & 257 & 43.63 & 0.94 \\
\hline Mean SF-12 Mental Health Score & 953 & 49.49 & 0.45 & 257 & 47.90 & 1.03 \\
\hline 6-Item Neurocognitive Score (continuous) & 805 & 5.46 & 0.03 & 244 & 5.33 & 0.07 \\
\hline Word fluency score (continuous) & 799 & 18.65 & 0.35 & 241 & 16.75 & 0.50 \\
\hline Digital symbol score (continuous) & 790 & 34.59 & 0.66 & 239 & 29.28 & 0.75 \\
\hline \multicolumn{7}{|l|}{ Self-reported chronic diseases } \\
\hline None & 474 & 48.16 & 2.05 & 41 & 14.61 & 2.64 \\
\hline One & 382 & 38.83 & 1.91 & 74 & 24.28 & 2.94 \\
\hline Two or more & 115 & 13.01 & 1.42 & 149 & 61.11 & 3.36 \\
\hline \multicolumn{7}{|l|}{ Smoking status } \\
\hline Never & 513 & 52.43 & 1.97 & 130 & 49.47 & 4.06 \\
\hline Former & 227 & 22.66 & 1.75 & 85 & 35.44 & 3.46 \\
\hline Current & 227 & 24.91 & 2.07 & 47 & 15.09 & 2.19 \\
\hline \multicolumn{7}{|l|}{ NIAAA risky alcohol use } \\
\hline Non-drinker & 335 & 39.69 & 2.17 & 116 & 57.37 & 4.39 \\
\hline Low-risk drinker & 385 & 53.87 & 2.23 & 69 & 38.63 & 4.54 \\
\hline At-risk drinker & 47 & 6.44 & 1.17 & 10 & 4.00 & 1.34 \\
\hline \multicolumn{7}{|l|}{ Meets 2008 physical activity guidelines } \\
\hline No & 420 & 43.41 & 1.79 & 136 & 56.59 & 3.42 \\
\hline Yes & 550 & 56.59 & 1.79 & 126 & 43.41 & 3.42 \\
\hline HbA1C (continuous) & 965 & 37.08 & 0.18 & 262 & 56.09 & 1.53 \\
\hline \multicolumn{7}{|l|}{ Self-reported visual impairment } \\
\hline Excellent/good & 493 & 53.23 & 2.06 & 147 & 58.13 & 4.43 \\
\hline Fair & 391 & 37.42 & 2.03 & 90 & 32.60 & 4.04 \\
\hline Poor/very poor & 86 & 9.34 & 1.18 & 26 & 9.27 & 1.96 \\
\hline NEI-VFQ composite score & 971 & 86.62 & 0.77 & 264 & 84.05 & 1.07 \\
\hline \multicolumn{7}{|l|}{ Ocular knowledge factors } \\
\hline \multicolumn{7}{|c|}{ Self-reported diabetes status at baseline examination } \\
\hline No & 961 & 98.91 & 0.40 & 117 & 41.34 & 3.39 \\
\hline Yes & 9 & 1.09 & 0.40 & 145 & 58.66 & 3.39 \\
\hline General eye knowledge summary scale & 971 & 6.88 & 0.06 & 264 & 6.92 & 0.12 \\
\hline \multicolumn{7}{|c|}{ Heard/seen something about eye health past 12 months from various sources } \\
\hline No sources & 292 & 29.96 & 1.89 & 68 & 27.67 & 3.26 \\
\hline One source & 362 & 35.52 & 1.96 & 94 & 34.98 & 3.44 \\
\hline Two or more sources & 317 & 34.52 & 2.28 & 100 & 37.36 & 3.53 \\
\hline \multicolumn{7}{|l|}{ Heard of diabetic retinopathy } \\
\hline Yes & 318 & 34.89 & 1.95 & 120 & 46.67 & 3.73 \\
\hline No & 652 & 65.11 & 1.95 & 143 & 53.33 & 3.73 \\
\hline
\end{tabular}

Ocular SOL, Ocular Study of Latinos; NEI-VFQ, National Eye Institute-Visual Functioning Questionnaire; SASH, Short Acculturation Score for Hispanics/Latinos; SE, standard error.

recommended by the healthcare provider because it is not possible to determine how many years a participant was living with undiagnosed diabetes, and the risk of diabetic retinopathy and increasingly severe, related complications increases with length of time living with diabetes. ${ }^{2}$ Kovarik et $a l^{66}$ found that although hospitalized patients (inpatients) had a higher prevalence of diabetic retinopathy than outpatients and that transportation and physical disability 
Table 2 Logistic regression models to show predictors of dilated eye examination within the past 12 months from the Ocular SOL (2011-2013)

\begin{tabular}{|c|c|c|c|c|c|c|}
\hline & $\begin{array}{l}\text { Model } 1 \\
\text { OR }(95 \% \mathrm{Cl})\end{array}$ & $\begin{array}{l}\text { Model } 2 \\
\text { OR (95\% Cl) }\end{array}$ & $\begin{array}{l}\text { Model } 3 \\
\text { OR (95\% Cl) }\end{array}$ & $\begin{array}{l}\text { Model } 4 \\
\text { OR (95\% Cl) }\end{array}$ & $\begin{array}{l}\text { Model } 5 \\
\text { OR (95\% Cl) }\end{array}$ & $\begin{array}{l}\text { Final model } \\
\text { OR }(95 \% \mathrm{Cl})\end{array}$ \\
\hline \multicolumn{7}{|l|}{ Predisposing factors (traditional) } \\
\hline \multicolumn{7}{|l|}{ Age group (years) } \\
\hline $40-54$ & REF & REF & REF & REF & REF & REF \\
\hline $55-64$ & 0.95 (0.48 to 1.86$)$ & 0.85 (0.39 to 1.82$)$ & 0.94 (0.37 to 2.38$)$ & $0.63(0.21$ to 1.87$)$ & 0.79 (0.38 to 1.65$)$ & 0.82 (0.40 to 1.65$)$ \\
\hline 65 and older & 4.24 (1.90 to 9.46$)$ & 5.83 (2.51 to 13.54$)$ & $3.02(1.27$ to 7.16$)$ & $1.35(0.43$ to 4.29$)$ & 2.43 (1.09 to 5.39$)$ & $2.62(1.22$ to 5.60$)$ \\
\hline \multicolumn{7}{|l|}{ Gender } \\
\hline Women & REF & REF & REF & REF & REF & REF \\
\hline Men & 1.34 (0.67 to 2.65$)$ & & & & & \\
\hline \multicolumn{7}{|l|}{ Hispanic Latino background } \\
\hline Cuban & REF & REF & REF & REF & REF & REF \\
\hline Central/South American & 0.80 (0.43 to 1.49$)$ & & & & & \\
\hline Other & 0.78 (0.18 to 3.34$)$ & & & & & \\
\hline \multicolumn{7}{|l|}{ Marital status } \\
\hline Single & REF & REF & REF & REF & REF & REF \\
\hline Married or living with partner & 1.05 (0.37 to 3.01$)$ & & & & & \\
\hline Separated/divorced/widowed & 0.91 (0.26 to 3.19$)$ & & & & & \\
\hline \multicolumn{7}{|l|}{ Educational attainment } \\
\hline No high school degree/GED & REF & REF & REF & REF & REF & REF \\
\hline High school degree/GED & $0.33(0.12$ to 0.91$)$ & 0.31 (0.10 to 0.95$)$ & $0.43(0.13$ to 1.40$)$ & 0.42 (0.08 to 2.26$)$ & $0.31(0.10$ to 1.00$)$ & $0.30(0.10$ to 0.96$)$ \\
\hline Greater than a high school degree/GED & 1.39 (0.63 to 3.05$)$ & $1.47(0.70$ to 3.09$)$ & $1.92(0.87$ to 4.23$)$ & $4.87(1.04$ to 22.79$)$ & $1.48(0.71$ to 3.11$)$ & 1.43 (0.69 to 2.98$)$ \\
\hline \multicolumn{7}{|l|}{ Employment status } \\
\hline Employed & REF & REF & REF & REF & REF & REF \\
\hline Retired, not currently employed & 1.59 (0.57 to 4.40$)$ & & & & & \\
\hline Unemployed & $1.32(0.67$ to 2.61$)$ & & & & & \\
\hline \multicolumn{7}{|l|}{ Predisposing factors (vulnerable) } \\
\hline \multicolumn{7}{|l|}{ Place of birth } \\
\hline $\begin{array}{l}\text { Foreign born } \\
\text { USA born (including territories) }\end{array}$ & REF & $\begin{array}{l}\text { REF } \\
0.99 \text { (0.08 to } 12.05)\end{array}$ & REF & REF & REF & REF \\
\hline \multicolumn{7}{|l|}{ Years lived in USA (if foreign born) } \\
\hline$\leq 5$ & REF & REF & REF & REF & REF & REF \\
\hline $6-15$ & & 0.76 (0.24 to 2.35$)$ & $1.16(0.36$ to 3.79$)$ & & & \\
\hline$>15$ & & $1.78(0.53$ to 5.98$)$ & $1.77(0.49$ to 6.31$)$ & & & \\
\hline SASH Language subscale & & 1.49 (0.39 to 5.72$)$ & & & & \\
\hline SASH Ethnic Social Relations subscale & & $1.23(0.52$ to 2.93$)$ & & & & \\
\hline Enabling factors & & & & & & \\
\hline \multicolumn{7}{|l|}{ Health insurance } \\
\hline Currently insured & REF & REF & REF & REF & REF & REF \\
\hline Currently uninsured & & & $0.49(0.18$ to 1.33$)$ & $0.27(0.07$ to 1.00$)$ & $0.37(0.15$ to 0.88$)$ & 0.34 ( 0.14 to 0.83 ) \\
\hline Never been insured & & & $0.37(0.11$ to 1.25$)$ & $0.11(0.03$ to 0.37$)$ & 0.21 (0.07 to 0.58$)$ & $0.19(0.07$ to 0.51$)$ \\
\hline
\end{tabular}




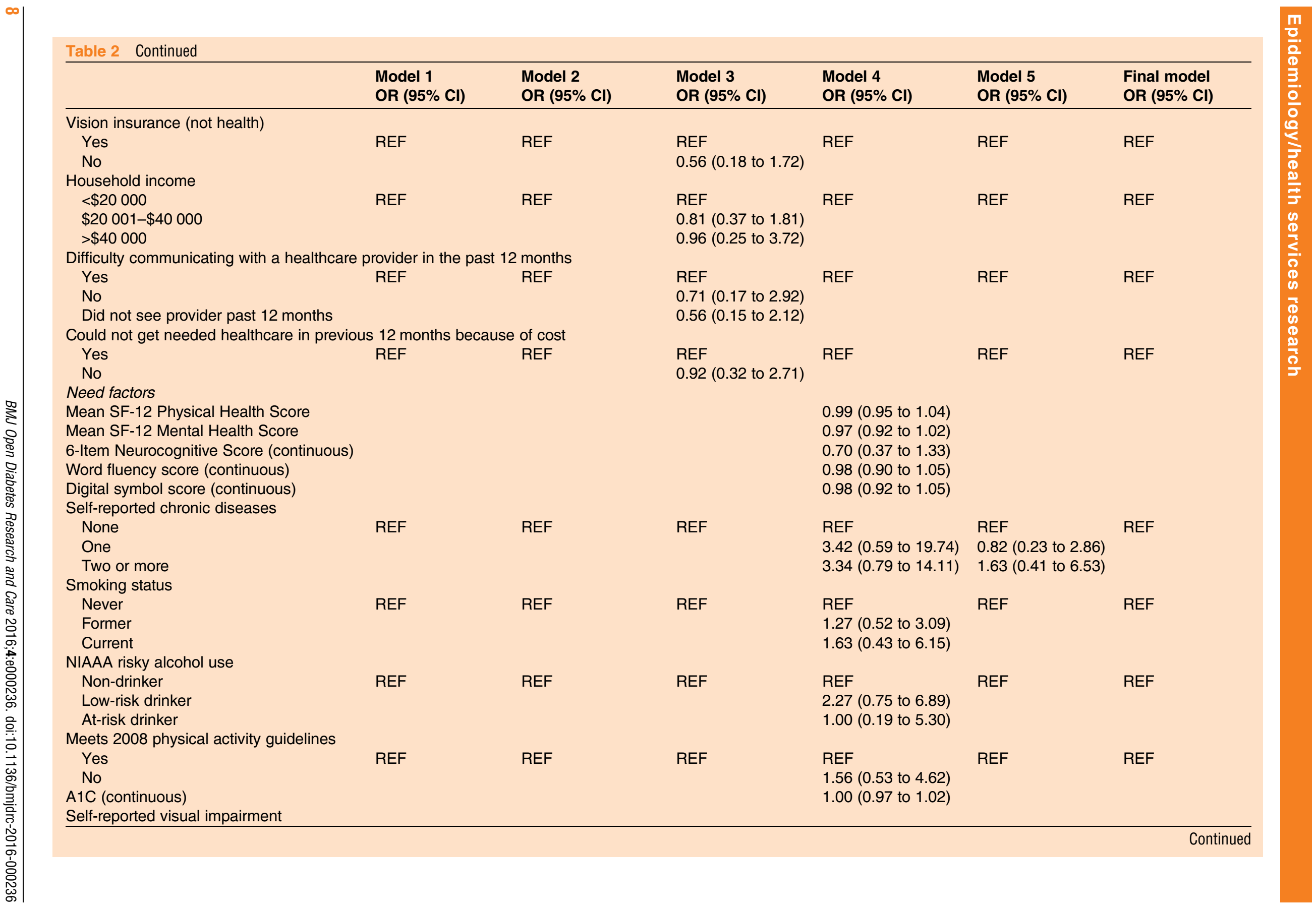




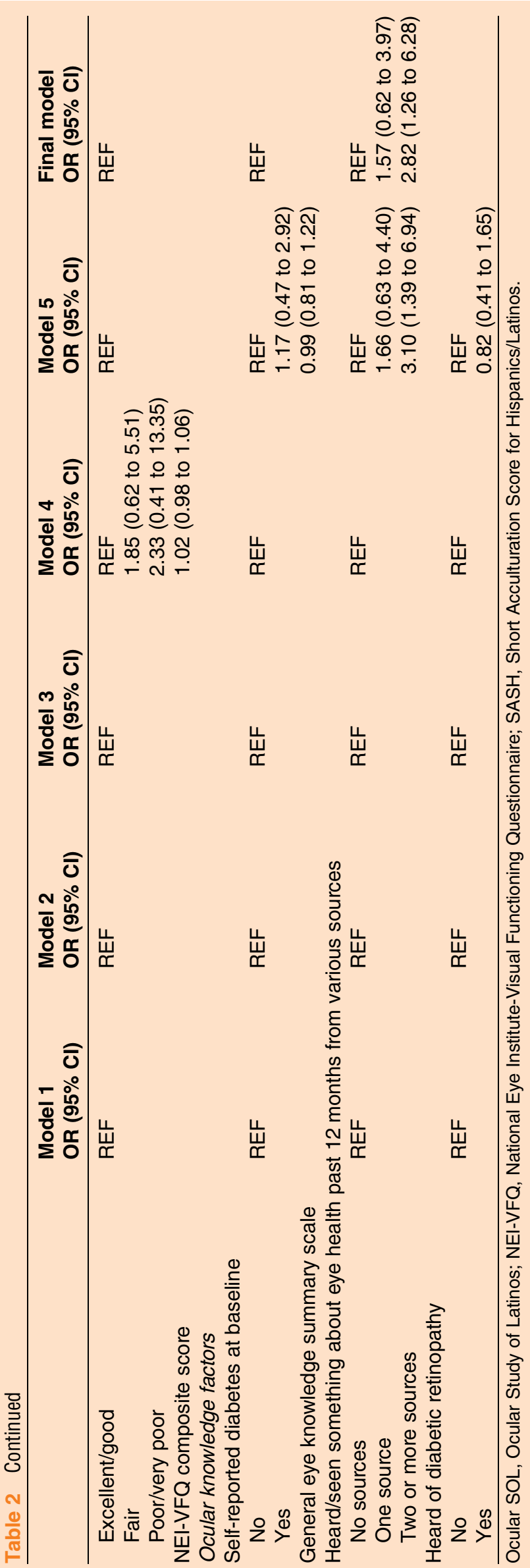

were barriers to eye care, they were more aware of ocular complications from having diabetes.

Our findings also demonstrate that only $53.3 \%$ of Hispanics/Latinos living with diabetes in our study had heard of diabetic retinopathy, suggesting that more than half may not have knowledge of the blinding, yet preventable, nature of diabetes. A study by Munoz et $a \hat{l}^{34}$ found that Hispanics/Latinos who had uncontrolled diabetes were less knowledgeable about secondary problems, such as diabetic retinopathy. Similar to our findings, these investigators found that only half of those living with diabetes for longer than 12 months knew that diabetes could cause eye disease. Finally, we found no appreciable difference in general eye knowledge by diabetes status (table 1). That is, one would expect that those with a diabetes diagnosis would have a higher knowledge score for eye health than those without diabetes and suggests that this group needs further targeting to improve their knowledge of their disease.

In our study, $13.0 \%$ and $18.7 \%$ of Hispanics/Latinos living with diabetes never had health insurance or were currently uninsured, respectively (table 1). Several additional demographic factors related to a diabetes diagnosis were consistent in our study with previous studies, ${ }^{23}{ }^{37}$ including a greater proportion of Hispanics/Latinos with diabetes who were employed, currently insured, and had less than a high school degree or GED compared to those without diabetes.

\section{Multivariable modeling of adherence with retinopathy screening guidelines among Hispanics/Latinos living with diabetes}

Multivariable sequential models identified only 4 out of 31 factors from Anderson's model and the Behavioral Model for Vulnerable Populations that were significant in predicting having a dilated eye examination in the past 12 months. We found age group to be associated with adherence to proper eye care for diabetes. Controlling for insurance status, Hispanics/Latinos with diabetes who were aged 65 years and older were more than 2.5 times likely to have a dilated eye examination within the past 12 months than those aged 40-54 years. In analysis of the entire HCHS/SOL cohort, Schneiderman et $a l^{6}$ found that older Hispanics/Latinos were more likely to be aware of having diabetes ( $p=0.0011)$, controlling for sex, education, years living in the USA, and Hispanic/Latino background. Similarly, in our analysis, having knowledge of diabetes may be the reason for older adults to be more adherent with the guidelines for dilated eye examination. Alternatively, it may be due to greater insurance coverage by Medicare in this older age group. Similarly, older age was associated with eye care in the past year in a sample of predominantly low income African-Americans. ${ }^{38}$

Health insurance status was strongly associated with having a dilated eye examination within the past year. Controlling for age and education, those currently uninsured were three times less likely to be adherent, while 
those never insured were more than five times less likely to be adherent. Our findings strongly suggest that not having insurance is an enormous barrier to receiving needed eye care in Hispanic/Latinos; targeted programs to deliver screening access to those in need are warranted.

Hispanics/Latinos with diabetes who heard about eye health from two or more sources were more likely to follow the recommendations for dilated eye screening than those who could recall no sources. Other researchers have found that interventions designed to increase adherence to diabetic retinopathy screening were mostly successful especially when the patient was aware of the risk involved with such a diagnosis. ${ }^{39}$ This raises the possibility that those who are aware of the secondary health problems associated with a diagnosis of diabetes may be paying closer attention to sources of information about eye health. The number of sources (or amount of exposure) is important, but so is the quality and accuracy of these sources of eye health information to increasing appropriate screening among those with diabetes. Weiss et $a l^{40}$ found that when older African-Americans with diabetes underwent a behavioral intervention providing sources of information such as educational pamphlets, online information, and ophthalmology contact information, they were more likely to have a dilated eye examination within the following 6 months compared to those not receiving the intervention. Primary healthcare providers must be a key source of eye health information to their patients with diabetes, and they must also actively refer and encourage their patients to seek annual exams.

Hispanics/Latinos in our study who had a high school diploma or GED were less likely to have an eye examination than those without a high school degree, and this unexpected finding was consistent throughout all models. Although those with greater than high school education reported that they were more likely to have an eye examination, the finding did not reach statistical significance. Categorization of the education variable may be an important factor explaining how our findings fit into the general literature. In the Los Angeles Latino Eye Study (LALES) study, participants with diabetes not completing high school were less likely to adhere to annual dilated eye examinations compared to those with a high school degree and more. ${ }^{20}$ In another LALES analysis of all study participants, in multivariable models while controlling for most of the same factors as in our study, compared to those Hispanics/Latinos of primarily Mexican heritage finishing grade 5 or less, only those with higher than high school education were more likely to have had at least one dilated eye examination in the past 12 months (OR 1.56, 95\% CI 1.21 to 1.99 ) ${ }^{23}$ Other investigators found that higher education, that is, high school degree and more, was non-significantly predictive of Hispanics/Latinos receiving a dilated eye examination within the past 2 years. ${ }^{34}$ Potential explanations for our seemingly counterintuitive finding are not clear. However, one explanation is inherent in the data collection of the baseline examination when participants were asked to indicate 'What was the highest grade/level of education achieved? If exact level is not listed, mark the closest equivalent'. There were three choices available: less than high school, high school or GED, or more than high school. Owing to the manner in which this question was asked, it is not possible to distinguish GED holders from those who earned a high school diploma. Previous research has indicated that those with a GED-only education have health status and health behavior patterns similar to non-high school rather than high school graduates. ${ }^{41}$ Since these other investigators did not break down education as we did (ie, less than high school, high school or GED, greater than high school), comparing our findings to theirs is difficult; this education finding needs to be further investigated in other studies.

We had several limitations in this study including a relatively small sample of participants living with diabetes. With the exception of marital status and not seeing a provider in the past 12 months, our sample was not significantly different from the rest of the HCHS/ SOL sample at the Miami site, ${ }^{25}$ but we are unable to extrapolate these findings to all Hispanics/Latinos living in the USA as our sample was largely comprised of participants of Cuban and Central/South American heritage. We also did not have power to distinguish between these Hispanic/Latino heritage groups. However, this unique sample of Hispanics/Latinos of Cuban heritage with diabetes serves to fill a gap in the literature as a majority of similar studies have been conducted largely among Hispanics/Latinos of Mexican heritage. ${ }^{23} 37$ Obtaining a dilated eye examination may require a referral from a doctor or healthcare organization, a practice variation that is outside of the control of the patient. The Ocular SOL study did not ask participants if they had difficulty obtaining any required referrals. Another important potential limitation is that accuracy of our self-reported dilated eye examination data in these Hispanic/Latino heritage groups is unknown. In fact, we could find no paper in the literature that documented the accuracy of self-reported eye examination in any Hispanic/Latino population group. Finally, owing to the cross-sectional design of our study, we can only speculate that viewing numerous eye health sources, for example, seeing advertisements for eye care on TV, in magazines, or on a billboard is influencing behavior to receive eye care. An alternate explanation for this association is that individuals who get an eye examination are simply more likely to recall having seen information relevant to ocular care. Interventions targeting Hispanic/Latino populations are needed to determine if greater information exposure actually leads to increased eye care utilization and which information sources are most effective at encouraging this behavior. When African-Americans with diabetes were given an educational intervention regarding the importance of eye care, the uptake of screening for diabetic retinopathy increased over the 
following 6 months compared to African-Americans who did not receive this treatment. ${ }^{40}$

In summary, health promotion and education strategies that stress the importance of annual dilated eye examinations among Hispanics/Latinos with diabetes may affect a change in the number of Hispanics/Latinos who are examined, ultimately decreasing the burden of visual impairment and blindness secondary to diabetic retinopathy. Healthcare providers should play a key role in educating their patients with diabetes of the need for annual examinations. The critical role of health insurance in enhancing adherence with retinopathy screening guidelines cannot be overemphasized. Particularly vulnerable are those who have never had health insurance. Safety-net programs for this segment of the population should be considered a high priority.

\section{Author affiliations}

${ }^{1}$ Sylvester Comprehensive Cancer Center, University of Miami Miller School of Medicine, Miami, Florida, USA

${ }^{2}$ Department of Public Health Sciences, University of Miami Miller School of Medicine, Miami, Florida, USA

${ }^{3}$ Department of Ophthalmology, Bascom Palmer Eye Institute, University of Miami Miller School of Medicine, Miami, Florida, USA

${ }^{4}$ Department of Ophthalmology and Visual Sciences, Division of Epidemiology and Biostatistics, School of Public Health, University of Illinois at Chicago, Chicago, Illinois, USA

${ }^{5}$ Division of Behavioral Sciences, Graduate School of Public Health, San Diego State University, San Diego, California, USA

Contributors SLT, DDZ, KLA and DJL analysed the data and wrote the manuscript, LAM, BLL wrote the manuscript, CEJ and GAT wrote and edited the manuscript, and SLT is the guarantor of this study.

Funding This research was supported by Centers for Disease Control and Prevention (5U58DP002651, 1U58DP002652, 5U58DP002653,

1U58DP002655). Grantees include the University of Alabama at Birmingham, Johns Hopkins University, Wills Eye Hospital, and the University of Miami. The HCHS/SOL was carried out as a collaborative study supported by contracts from the National Heart, Lung, and Blood Institute (NHLBI) to the University of North Carolina (N01-HC65233), University of Miami (N01-HC65234), Albert Einstein College of Medicine (N01-HC65235), Northwestern University (N01-HC65236), and San Diego State University (N01-HC65237). The following Institutes/Centers/Offices contribute to the HCHS/SOL through a transfer of funds to the NHLBI: National Institute on Minority Health and Health Disparities, National Institute on Deafness and Other Communication Disorders, National Institute of Dental and Craniofacial Research, National Institute of Diabetes and Digestive and Kidney Diseases, National Institute of Neurological Disorders and Stroke, and $\mathrm{NIH}$ Institution-Office of Dietary Supplements. The funding organizations had no role in the design or conduct of this research.

Competing interests None declared.

Ethics approval This study was approved by University of Miami, Miami, Florida, USA.

Provenance and peer review Not commissioned; externally peer reviewed.

Data sharing statement No additional data are available.

Open Access This is an Open Access article distributed in accordance with the Creative Commons Attribution Non Commercial (CC BY-NC 4.0) license, which permits others to distribute, remix, adapt, build upon this work noncommercially, and license their derivative works on different terms, provided the original work is properly cited and the use is non-commercial. See: http:// creativecommons.org/licenses/by-nc/4.0/

\section{REFERENCES}

1. Cheng YJ, Imperatore G, Geiss LS, et al. Secular changes in the age-specific prevalence of diabetes among U.S. adults: 1988-2010. Diabetes Care 2013;36:2690-6.

2. Antonetti DA, Klein R, Gardner TW. Diabetic retinopathy. N Engl J Med 2012;366:1227-39.

3. American Academy of Ophthalmology Preferred Practice Pattern Guidelines-Diabetic Retinopathy PPP-Updated October 2014. http://www.aao.org/preferred-practice-pattern/ diabetic-retinopathy-ppp--2014 (accessed 10 Feb 2016).

4. American Diabetes Association. Standards of medical care in diabetes-2013. Diabetes Care 2013;36(Suppl 1):S11-66.

5. Bragge $P$, Gruen RL, Chau M, et al. Screening for presence or absence of diabetic retinopathy: a meta-analysis. Arch Ophthalmol 2011;129:435-44.

6. Schneiderman N, Llabre M, Cowie CC, et al. Prevalence of diabetes among Hispanics/Latinos from diverse backgrounds: the Hispanic Community Health Study/Study of Latinos (HCHS/SOL). Diabetes Care 2014;37:2233-9.

7. Centers for Disease Control and Prevention. ed. National diabetes fact sheet: national estimates and general information on diabetes and prediabetes in the United States. Atlanta, GA: U.S. Department of Health and Human Services, Centers for Disease Control and Prevention, 2011.

8. Centers for Disease Control and Prevention. National Diabetes Statistics Report. 2014. http://www.cdc.gov/diabetes/pubs/ statsreport14/national-diabetes-report-web.pdf (accessed 10 Feb 2016).

9. Varma R, Torres M, Pena F, et al. Prevalence of diabetic retinopathy in adult Latinos: the Los Angeles Latino Eye Study. Ophthalmology 2004;111:1298-306.

10. Gao X, Gauderman W, Marjoram P, et al. Genetic variants associated with proliferative diabetic retinopathy in Latinos. Invest Ophthalmol Vis Sci 2014;55:2238.

11. Gonzalez Burchard E, Borrell LN, Choudhry S, et al. Latino populations: a unique opportunity for the study of race, genetics, and social environment in epidemiological research. Am J Public Health 2005;95:2161-8.

12. Elder JP, Ayala GX, Parra-Medina D, et al. Health communication in the Latino community: issues and approaches. Annu Rev Public Health 2009;30:227-51.

13. Carabellese C, Appollonio I, Rozzini R, et al. Sensory impairment and quality of life in a community elderly population. J Am Geriatr Soc 1993;41:401-7.

14. Reuben DB, Mui S, Damesyn M, et al. The prognostic value of sensory impairment in older persons. J Am Geriatr Soc 1999;47:930-5.

15. Appollonio I, Carabellese C, Magni E, et al. Sensory impairments and mortality in an elderly community population: a six-year follow-up study. Age Ageing 1995;24:30-6.

16. Wang JJ, Mitchell P, Simpson JM, et al. Visual impairment, age-related cataract, and mortality. Arch Ophthalmol 2001;119:1186-90.

17. Thompson JR, Gibson JM, Jagger C. The association between visual impairment and mortality in elderly people. Age Ageing 1989;18:83-8

18. McCarty CA, Nanjan MB, Taylor HR. Vision impairment predicts 5 year mortality. Br J Ophthalmol 2001;85:322-6.

19. Wallhagen MI, Strawbridge WJ, Shema SJ, et al. Comparative impact of hearing and vision impairment on subsequent functioning. J Am Geriatr Soc 2001;49:1086-92.

20. Paz SH, Varma R, Klein R, et al. Noncompliance with vision care guidelines in Latinos with type 2 diabetes mellitus: the Los Angeles Latino Eye Study. Ophthalmology 2006;113:1372-7.

21. Rodriguez J, Sanchez R, Munoz B, et al. Causes of blindness and visual impairment in a population-based sample of U.S. Hispanics. Ophthalmology 2002;109:737-43.

22. Varma R, Ying-Lai M, Klein R, et al. Prevalence and risk indicators of visual impairment and blindness in Latinos: the Los Angeles Latino Eye Study. Ophthalmology 2004;111:1132-40.

23. Morales LS, Varma R, Paz SH, et al. Self-reported use of eye care among Latinos: the Los Angeles Latino Eye Study. Ophthalmology 2010;117:207-15.e1.

24. Lavange LM, Kalsbeek WD, Sorlie PD, et al. Sample design and cohort selection in the Hispanic Community Health Study/Study of Latinos. Ann Epidemiol 2010;20:642-9.

25. McClure LA, Zheng DD, Lam BL, et al. Factors Associated with Ocular Healthcare Utilization among Hispanics/Latinos: results from the Ocular SOL Ancillary to the Hispanic Community Health Study/ Study of Latinos. JAMA Ophthal 2016;134:320-9. 
26. American Diabetes Association. Diagnosis and classification of diabetes mellitus. Diabetes Care 2010;33(Suppl 1):S62-69.

27. Andersen RM. Revisiting the behavioral model and access to medical care: does it matter? J Health Soc Behav 1995;36:1-10.

28. Gelberg L, Andersen RM, Leake BD. The behavioral model for vulnerable populations: application to medical care use and outcomes for homeless people. Health Serv Res 2000;34:1273-302.

29. Marin G, Sabogal F, Marin B, et al. Development of a short acculturation scale for Hispanics. Hisp J Behav Sci 1987;9:183-205.

30. Mangione CM, Lee PP, Gutierrez PR, et al. Development of the 25-item National Eye Institute Visual Function Questionnaire. Arch Ophthalmol 2001;119:1050-8.

31. Ware J Jr, Kosinski M, Keller SD. A 12-Item Short-Form Health Survey: construction of scales and preliminary tests of reliability and validity. Med Care 1996;34:220-33.

32. Gonzalez HM, Tarraf W, Gouskova N, et al. Neurocognitive function among middle-aged and older Hispanic/Latinos: results from the Hispanic Community Health Study/Study of Latinos. Arch Clin Neuropsychol 2015;30:68-77.

33. McKean-Cowdin R, Varma R, Hays RD, et al. Longitudinal changes in visual acuity and health-related quality of life: the Los Angeles Latino Eye study. Ophthalmology 2010;117:1900-7.

34. Munoz B, O'Leary M, Fonseca-Becker F, et al. Knowledge of diabetic eye disease and vision care guidelines among Hispanic individuals in Baltimore with and without diabetes. Arch Ophthalmol 2008;126:968-74.

35. Rowe $\mathrm{S}$, MacLean $\mathrm{CH}$, Shekelle PG. Preventing visual loss from chronic eye disease in primary care. JAMA 2004;291:

1487-96.

36. Kovarik JJ, Eller AW, Willard LA, et al. Prevalence of undiagnosed diabetic retinopathy among inpatients with diabetes: the diabetic retinopathy inpatient study (DRIPS). BMJ Open Diabetes Res Care 2016;4:e000164.

37. Mier N, Wang X, Smith ML, et al. Factors influencing health care utilization in older Hispanics with diabetes along the Texas-Mexico border. Popul Health Manag 2012;15:149-56.

38. Maclennan PA, McGwin G Jr, Heckemeyer C, et al. Eye care use among a high-risk diabetic population seen in a public hospital's clinics. JAMA Ophthalmol 2014:132:162-7.

39. Zhang X, Norris SL, Saadine J, et al. Effectiveness of interventions to promote screening for diabetic retinopathy. Am J Prev Med 2007:33:318-35.

40. Weiss DM, Casten RJ, Leiby BE, et al. Effect of behavioral intervention on dilated fundus examination rates in older African American individuals with diabetes mellitus: a randomized clinical trial. JAMA Ophthalmol 2015;133:1005-12.

41. Zajacova A. Health in working-aged Americans: adults with high school equivalency diploma are similar to dropouts, not high school graduates. Am J Public Health 2012;102(Suppl 2): S284-90. 